\title{
PREDICTION OF EARLY NEONATAL INFECTION IN PREGNANCIES WITH PRELABOUR RUPTURE OF MEMBRANES
}

\author{
Vlora Ademi Ibishi ${ }^{1}$ Rozalinda Dusan Isjanovska ${ }^{2}$ \\ ${ }^{1}$ University Clinical Center Of Kosova,Obstetrics and Gynecology Clinic,Prishtina,1000,Kosova \\ drvlora@hotmail.com \\ ${ }^{2}$ Institutefor Epidemiology and Medical Biostatistics,Medical Faculty,Ss. Cyril and Methodius University of \\ Skopje,Skopje,Republic of Macedonia \\ prof.isjanovska@gmail.com
}

\begin{abstract}
Background: The uncertainty of predicting which pregnancies will have early onset neonatal infection (EONI) with prelabour rupture of membranes (PROM) is a challenge to obstetricians because presence of this condition impacts the decision to do expectant or active management of the case. The most common maternal markers of infection used in our practice are C-reactive protein, white blood cell count, and amniotic fluid glucose concentration.
\end{abstract}

Objective: The primary objective of the study is to evaluate the accuracy of measuring C-reactive protein and leukocytes in maternal serum and amniotic fluid glucose concentration to predict early onset neonatal infection. The secondary objective of the study is to determine maternal fetal outcome in pregnancies with prelabour rupture of membranes.

Material and methods: This prospective cross-sectional study enrolled 170 pregnant patients who experienced prelabour rupture of chorioamniotic membranes.. The maternal serum and amniotic fluid samples were taken at admission and later used to analyze whether/how the test results were a predictor of early onset neonatal infection.

Results: C-reactive protein concentrations were significantly associated with early onset neonatal infection. Maternal CRP had $92.0 \%$ sensitivity and specificity of $62.8 \%$ for predicting early onset neonatal infection. Maternal serum white blood cell count and amniotic fluid glucose concentrations estimation after pre-labour rupture of membranes showed poor predictive value in neonatal early onset infection.

Conclusion: C-reactive protein was more sensitive in predicting Early Onset Neonatal Infection than either white blood cell count or amniotic fluid glucose concentration.

Key words: Early onset neonatal infection, maternal markers, pre-labour rupture of membranes

\section{Academic Discipline And Sub-Disciplines}

Medical Science

\section{SUBJECT CLASSIFICATION}

Obstetrics and Gynecology

Author Biography

Vlora Ademi Ibishi

Medical Faculty,University

of Prishtina,Kosovo

\section{Council for Innovative Research}

Peer Review Research Publishing System

\section{Journal: International Journal of Research in Education methodology}

\section{Vol. 7, No.2}

ijremeditor@gmail.com

www.ijrem.com 


\section{Background:}

Prelabour rupture of membranes (PROM) refers to rupture of the chorioamniotic membranes prior to the onset of labor and prior to the onset of clinically apparent labour contraction. PROM can occur at any gestational age. ${ }^{1}$ This condition is classified as "preterm PROM"if the event occurs before 37 weeks of gestation, or "term PROM" if the event occurs after 37 weeks gestation.. $2,3,4,5$ A numbers of factors have been associated with PROM including anatomic and pregnancy-related infection. ${ }^{6}$ Infection is an important known risk-factor associated with PROM. There is ongoing debate in the medical literature whether intrauterine infection is a cause or a consequence of pre-labor rupture of the fetal membranes. ${ }^{7}$ In both cases, subclinical infection may be present before and after PROM and is an etiologic factor in maternal-fetal consequences.

The most frequent maternal consequences associated with intra amniotic infection are endomyometritis, wound infection, pelvic abscess, bacteremia and postpartum haemorrhage. ${ }^{8,9}$

Fetal exposure to infection may lead to numerous adverse outcomes. Early Onset Neonatal Infection-EONI, generally acquired prenatally in pregnancies with PROM, is one of the most serious consequence of maternal infection and is associated with increased neonatal morbidity and mortality. ${ }^{10}$

Early identification of EONI is a desirable clinical goal because late diagnosis with delayed treatment increases neonatal morbidity and mortality. Despite numerous studies focusing on finding efficient markers as predictors of EONI, prediction of EONI remains a critical challenge in pregnancies with PROM ${ }^{11}$. Cost, availability of specimens at the appropriate time, complexity of the assay methods, laboratory turnover time, reliability of the tests, and attitude of attending clinicians are all important factors in determining the suitability of a diagnostic marker for clinical application ${ }^{12}$. The most common maternal markers used in practice are C-reactive protein, white blood cell count and glucose. Recent studies have focused on determining the accuracy of white blood cell count, CRP and glucose concentration separately or in combination for prediction of early onset neonatal infection in pregnancies complicated with PROM, but the results remain controversial.

Objective: The main objective of this research is to investigate the predictive value for EONI of C-reactive protein and leukocyte count in maternal serum and glucose concentration in amniotic fluid in pregnant women with PROM.

Secondary objectives of the study aim to determine maternal fetal outcome in pregnancies with pre-labour rupture of membranes.

Ethics: This study was submitted to the Institutional Ethical Review Committee and was found to be in conformity with the laws and regulations of the country in which the research was conducted. Written informed consent was obtained from all subjects. To maintain participant confidentiality numbers instead of names were used on the questionnaire and evaluation forms.

Material and Methods: This prospective cross-sectional study was conducted from June 2013 through March 2015 in Obstetrics and Gynecology Clinic of University Clinical Center of Kosova and investigated the predictive value for EONI of CRP, WBC and glucose concentration in pregnant women with PROM. One hundred and seventy eight pregnant patients between 24-41 weeks of gestational age who were not on either antibiotic or corticoid treatment and who experienced pre-labour rupture of amniotic membranes were assessed for eligibility. Of these 178 patients, 8 pregnant women were excluded due to hypertensive disorders, diabetes mellitus, fetal malformations and giving birth beyond 72 hours after admission.. A total of 170 pregnant women and their newborns $(\mathrm{N}=170)$ were then eligible to be included in the study.

Study methods of intervention and data collection included a specific questionnaire and evaluation form used to collect data prospectively at admission and thereafter. Data covering demographic, maternal and neonatal characteristics were recorded and analyzed.

PROM was defined as leakage of amniotic fluid that precedes the onset of uterine contractions and the cervical canal dilatation of less than $2 \mathrm{~cm}$. Confirmation of the diagnosis of rupture of membranes was documented by sterile speculum examination confirming the pooling of amniotic fluid in the posterior vaginal fornix or/and direct visualization of fluid leakage from the cervical canal. Maternal serum and amniotic fluid samples were taken at the admission from all the women.

About two milliliters of blood was collected by venipuncture. .Blood samples were transported to the biochemistry department for total WBC (expressed as cells $\times 10^{9}$ ) and CRP (expressed as $\mathrm{mg} / \mathrm{L}$ ) estimation. CRP levels were estimated using a latex agglutination test. The pathological limit for leukocyte count was considered value $\geq 14 \times 10^{9}$ and for CRP value $>6 \mathrm{mg} / \mathrm{L}$. Three milliliters of amniotic fluid were collected vaginally and samples are used to measure the glucose concentration (expressed as $\mathrm{mg} / \mathrm{dl}$ ). Pathological amniotic fluid glucose concentration was considered value $\leq 14 \mathrm{mg} / \mathrm{dl}$.

\section{Outcome definition:}

Immediately after the delivery all newborns were observed for complications related to infectious morbidity. The newborns were observed during the first seven days of life respectively during early neonatal period. Neonatal infection was considered probable based on clinical signs and a neonatal CRP $\geq 10 \mathrm{mg} / \mathrm{dl}$ and thereafter was confirmed as indicated by radiography, positive blood culture or a positive cerebrospinal fluid culture associated with clinical signs of infection.

\section{Statistical analysis:}

Statistical analysis was performed by using statistical package SPSS 17.0 version software. The data are expressed in number and percentage, as well as, in average and standard deviation. The $95 \%$ of $\mathrm{Cl}$ was also calculated. For determining the the accuracy of markers as diagnostic methods, Sensitivity, Specificity, Positive Predictive Value, Negative Predictive Value and Likelihood ratio were calculated. Statistical significance was defined as $p-v a l u e<0.05$. 


\section{Results:}

One hundred and seventy pregnant women with a diagnosis of premature rupture of membranes and their newborns $(n=170)$ were included in this study. The clinical characteristics of the study participants are presented in Table 1 . The average age of the pregnant women involved in the research was 27.4 years old (SD \pm 5.4 years). The majority of the participants were nulliparous (65,8\%). Among all patients, $26.7 \%(\mathrm{~N}=8)$ had previous abortions, $3.35 \%(\mathrm{~N}=6) \mathrm{had}$ previous uterus surgery, and $25.2 \%(\mathrm{~N}=43)$ were smokers. Mean gestational age at birth was 37.4 weeks (SD \pm 2.52 weeks) and the mean time interval between PROM and delivery was 24.4 hours (SD $\pm 14.52 \mathrm{hrs).} \mathrm{EONI} \mathrm{was} \mathrm{diagnosed} \mathrm{in}$ 25 cases respectively in $14.7 \%$ of newborns. The average weight of newborns was 3064 gram (SD $\pm 675.2 \mathrm{gram}$ ). Twenty seven and a half percent $(\mathrm{N}=46)$ of the 170 study participants delivered by cesarean section and the remaining $72.5 \%(\mathrm{~N}=124)$ delivered vaginally.

Table 1. Maternal and Neonatal Characteristics and Mode of Delivery

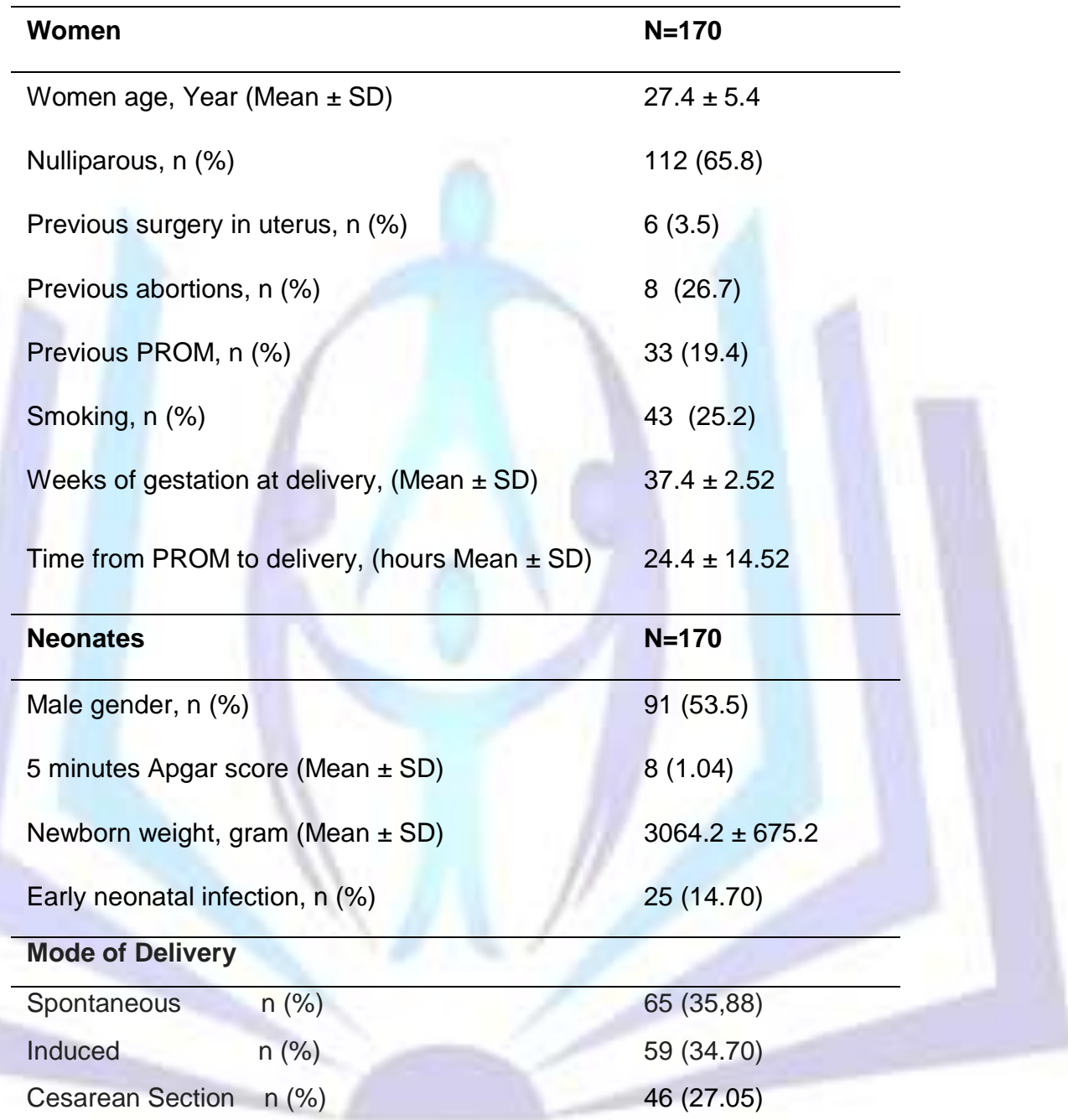

Pre-labour Rupture of Membranes is an important cause of maternal and fetal morbidity. Postpartum hemorrhage and retained placenta are most common maternal adverse outcomes observed in our study. EONI was present in 25 cases. In total, 2 neonatal deaths were registered in the group of newborns with confirmed early neonatal sepsis. Neonatal infectious morbidity and other maternal adverse outcomes are presented in Table 2.

Table 2. Maternal / Neonatal Outcome in Pregnancies Complicated with PROM

Maternal morbidity / complications

\begin{tabular}{lccc}
\hline & Count & Percent & Cumulative \\
No complications & 158 & 92,94118 & 92,9412 \\
Chorioamnionitis & 2 & 1,17647 & 94,1176 \\
PP haemorrhage & 3 & 1,76471 & 95,8824 \\
Placental abruption & 1 & 0,58824 & 96,4706 \\
Retained placenta & 3 & 1,76471 & 98,2353
\end{tabular}


Pelvic abscess

Endometritis

Wound infection
1

1

1
0,58824

0,58824

0,58824
98,8235

99,4118

100,0000

Early Neonatal Outcome/ Infection

\begin{tabular}{lccr}
\hline No complications & 145 & 85,29411 & 85,2941 \\
Clinical infection & 3 & 1,76470 & 87,0588 \\
SIRS & 4 & 2.35294 & 89,4117 \\
Pneumonia & 8 & 4,70588 & 94,1176 \\
Sepsis & 10 & 5,88235 & 100.0000
\end{tabular}

In order to verify the hypothesis, which is that serum CRP and WBC and amniotic fluid glucose may serve as a marker for prediction of EONI in pregnancies with PROM, the newborns were divided into two groups. Group I was newborns with no infection ( $\mathrm{N}=145)$. Group II was, newborns with early onset neonatal infections $(\mathrm{N}=25)$. The average value of CRP at mothers who gave birth to newborns of the group with neonatal infections was much higher, a difference of significant statistical importance $(\mathrm{P}<0.0000)$. The average value of WBC at mothers of Group II of newborns with neonatal infections was a bit higher but without a significant difference $(P>0.05)$. Value of glucoses in amniotic fluid at mothers of Group II of newborns with neonatal infections was a bit higher but with no significant difference $(P>0.05)$. (Table 3$)$

Table 3. Mean Value of CRP, WBC and Glucose by Groups

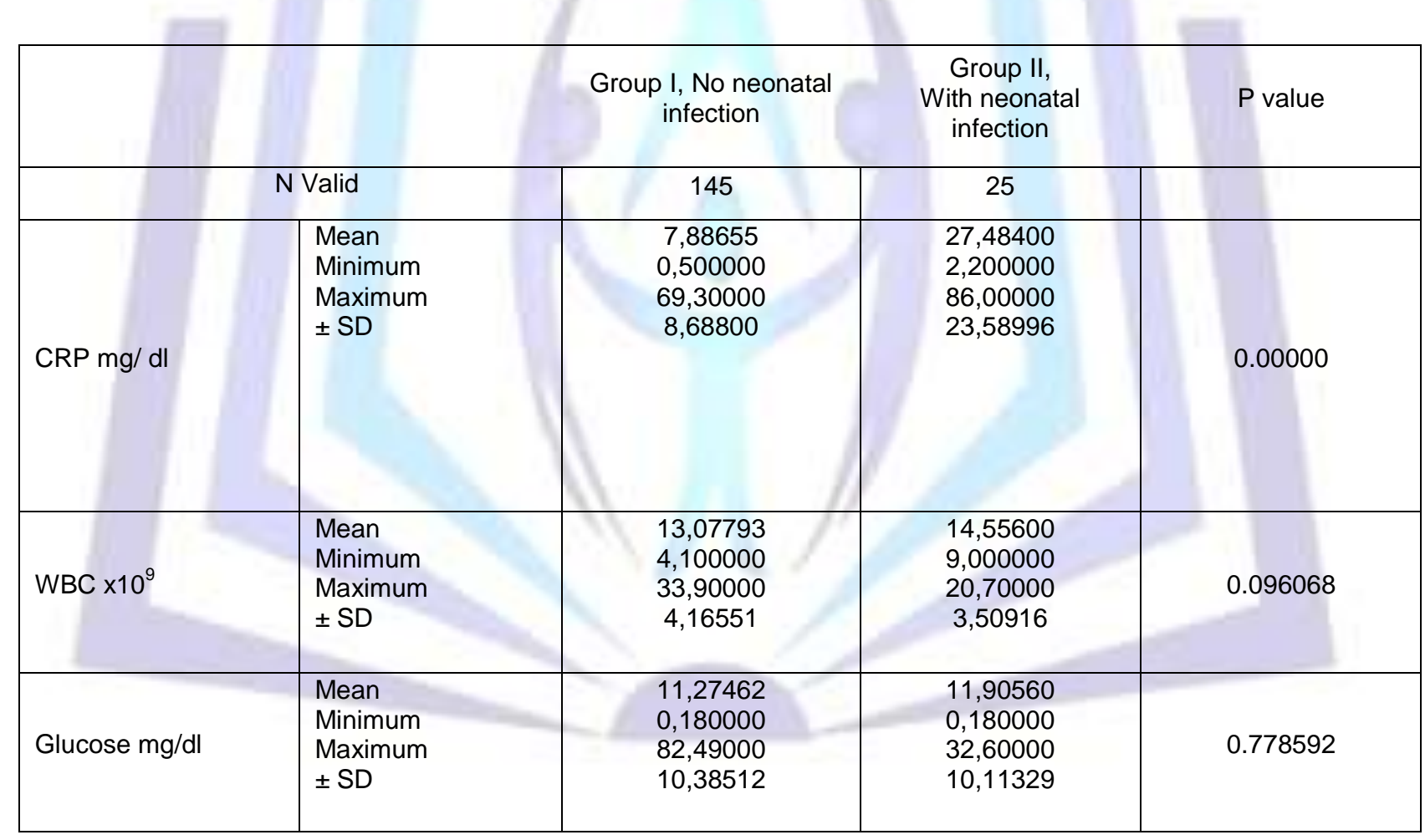

The results in this study report a high sensitivity of CRP for prediction of EONI in pregnancies with PROM, respectively $92.0 \%(95 \% \mathrm{Cl} 75.0-97.8)$ and likehood ratio of 2.44. WBC and glucose do not have significant sensitivity in prediction of EONI in pregnancies with PROM (Table 4). 
Table 4. Sensitivity and specificity of CRP, WBC and Glucose on prediction of EONI

\begin{tabular}{llllllll}
\hline & CRP & {$[95 \% \mathrm{Cl}]$} & WBC & {$[95 \% \mathrm{Cl}]$} & Glucose & [95 \% Cl] \\
\hline Sensitivity & 0.92 & {$[0.75$ to 0.978$]$} & 0.6 & {$[0.407$ to 0.766$]$} & 0.76 & [0.566 to 0.885$]$ \\
Specificity & 0.628 & {$[0.547$ to 0.702$]$} & 0.676 & {$[0.596$ to 0.747$]$} & 0.269 & [ 0.203 to 0.346$]$ \\
PPV & 0.299 & {$[0.208$ to 0.408$]$} & 0.242 & {$[0.152$ to 0.362$]$} & 0.152 & [0.1 to 0.225$]$ \\
NPV & 0.978 & {$[0.925$ to 0.994$]$} & 0.907 & {$[0.838$ to 0.949$]$} & 0.867 & [ 0.738 to 0.937$]$ \\
LR+ & 2.47 & {$[1.942$ to 3.143$]$} & 1.851 & {$[1.244$ to 2.753$]$} & 1.04 & {$[0.817$ to 1.323$]$} \\
LR- & 0.127 & {$[0.034$ to 0.484$]$} & 0.592 & {$[0.361$ to 0.969$]$} & 0.892 & {$[0.423$ to 1.884$]$}
\end{tabular}

Discussion: Leading causes of early neonatal death globally are complications of preterm birth, intra partum related causes and infection. In Kosova, despite the reported decrease, the early neonatal mortality rate remains high compared to other European countries. Infection continues to pose a problem and is implicated in the pathogenesis of pre-labour rupture of membranes. The link between infectious early neonatal morbidity and mortality and pre-labour rupture of membranes is incontestable. It is accepted that women with complicated with pre-labour rupture of membranes have a substantially higher risk of neonatal morbidity and mortality due to infection than women without membrane ruptures. ${ }^{14}$

Prediction of early onset neonatal infection in these cases remains a major challenge and there is no decisive standard protocol on what are the best methods to use. Medical literature reports different invasive and non-invasive methods and markers to predict EONI in pregnancies complicated with PROM. Cost, availability of specimens at the appropriate time, complexity of the assay methods, laboratory turnover time, reliability of the tests, and attitude of attending clinicians are all important factors in determining the suitability of a diagnostic marker for clinical application. ${ }^{15}$

The existing medical research on the accuracy of different maternal markers for prediction of EONI in pregnancies complicated with PROM remains unclear. Different studies have identified different markers as accurate predictors of EONI/PROM and so there is not a consensus of what test is best to use. The paragraphs below summarize existing literature on identifying accurate predictors.

Several studies reported the accuracy of CRP as a predictive marker including Sung Youn Lee and al (2012). In in their study about relationship between maternal serum CRP and early onset neonatal sepsis, they reported success in finding a good negative predictive value in excluding early-onset neonatal sepsis. Yee et al .reported that that maternal serum CRP level $<8 \mathrm{mg} / \mathrm{L}$ had a good negative predictive value in excluding early-onset neonatal sepsis; therefore, this test has potential to be a useful non-invasive adjunct to clinical judgment to identify low-risk patients ${ }^{16}$.

Popowski et al.(2011) also concluded that CRP at admission was an accurate marker for predictin EONI. ${ }^{17}$ These researchers conducted an investigation in France of maternal markers for detecting EONI and chorioamnionitis in cases of premature rupture of membranes at or after 34 weeks of gestation. In their study of 399 woman with PROM, maternal serum CRP at admission is the most accurate infectious marker for predicting EONI Park et Al (2012) studied the accuracy of measuring CRP and leukocytes in maternal serum to predict the probability of intra-amniotic infection among 171 women with PPROM. In addition to CRP, the study reported that WBC, parity, and gestational age are also highly predictive of IAI in women with PPROMs ${ }^{18}$.

Buhimschi et al (2006) also investigated the predictive value for EONI of $C$ reactive protein, white blood cell count, and glucose concentration and showed that maternal serum CRP at admission is the most accurate infectious marker for predicting EONI.

By ROC curve, the sensitivity and specificity of maternal CRP for early onset neonatal infection in our data was $92 \%$ and $68 \%$ respectively. Investigation of maternal WBC and amniotic fluid glucose concentration showed low sensitivity in prediction of EONI.

This study also reported that glucose concentration as a marker used to diagnose infection in other sites such as cerebrospinal fluid was not a good predictor. Vaginal glucose determination is a readily available, inexpensive, rapid AF marker that can be measured practically in any clinical laboratory. Buhimschi et al (2006) investigated the value of low vaginal pool glucose measurement for prediction of intra amniotic infection. The results showed that vaginal amniotic fluid glucose measurements less than $15 \mathrm{mg} / \mathrm{dL}$ have predictive value, but low sensitivity for detection of $\mid \mathrm{AI}{ }^{20}$.

Van der Heyden et al (2010), however, did not support the use of CRP. In their study, they researched the accuracy of measuring CRP and leukocytes in maternal serum to predict neonatal infection among 299 women with PROM. The results showed that in women with PROM, CRP and leukocytes should not be measured routinely. ${ }^{19}$ 


\section{Conclusion:}

Prelabour Rupture of Membranes remains an important cause of maternal and fetal morbidity and increased rate of cesarean section delivery. Postpartum haemorrhage, retained placenta and chorioamnionitis are the most common maternal complicatio. Neonatal infection related to PROM is an important factor of neonatal morbidity especially in pre term born infants.

CRP, WBC count and Glucose concentration are the most common markers of infection used in practice. This study found that in cases with pre-labour rupture of membranes, maternal CRP is a more accurate predictor of EONI in pregnancies with PROM than WBC and glucose concentration. These study results contribute to the ongoing research question of determining the accuracy of infection markers and the results add to the growing number of studies that report CRP as an accurate predictor of EONI.

\section{References:}

1. Keirse MJ, Ohlsson A, Treffers PE, Kanhani HHH. Prelabour rupture of the membranes preterm. In: Chalmers I, Enkin M, Keirse MJ, eds. Effective care in pregnancy and childbirth.Oxford: Oxford University Press; 1989:666.

2. Verber IG, Pearce JM, New LC, Hamilton PA, Davies EG. Prolonged rupture of the fetal membranes and neonatal outcome. J Perinat Med 1989; 17:469-76.

3. Mercer BM. Preterm premature rupture of the membranes. Obstet Gynecol 2003; 101:178-93

4. Tamsen L, Lyrenas S, Cnattingius S. Premature rupture of the membranes-intervention or not. Gynecol Obstet Invest 1990; 29:128-31

5. Grant J, Keirse MJNC: Prelabour rupture of the membranes at term. In Effective Care in Pregnancy and Childbirth. Edited by Chalmers I, Enkin M and Keirse MJNC. Oxford, Oxford University Press; 1989:1112-1117

6. Current Clinical Practice: Obstetrics in Family Medicine: A Practical Guide. P. Lyons (C) Humana Press Inc., Totowa, New Jersey, 2006

7. Samuel Parry,M.D. and Jerome F.Strauss III,M.D, PhD. Premature rupture of the fetal membranes :Mechanism of disease, Kofinas Perinatal Providing Care to the Unborn ${ }^{\circledR}$

8. Rouse DJ, Landon M, Leveno KJ, et al. The Maternal-Fetal Medicine Units cesarean registry: chorioamnionitis at term and its duration-relationship to outcomes. Am J Obstet Gynecol 2004;191:211

9. Mark SP, Croughan-Minihane MS, Kilpatrick SJ. Chorioamnionitis and uterine function. Obstet Gynecol 2000;95:909

10. Cohen-Wolkowiez $M$ et al: Early and late onset sepsis in late preterm infants. The Pediatric infectious disease journal 2009, 28(12):1052-1056.

11. Gotoff SP. Infection in neonatal infant. In: Behrman RE,Kliegman RM, Jenson HB, editors. Nelson's Textbook of Pediatrics. 16th edition. Philadelphia (PA): WB SaundersCo; 2000. p. 938-952

12. P C Ng, Diagnostic markers of infection in neonates, Arch Dis Child Fetal Neonatal Ed 2004;89:F229-F235

13. Population Division.Levels and Trends in Child Mortality-Report 2012, UNICEF 2012

14. Cobo T et Al. Umbilical cord blood IL-6 as predictor of early-onset neonatal sepsis in women with preterm prelabour rupture of membranes.PloS One. 2013 Jul 24;8(7)

15. $\mathrm{P} \mathrm{C} \mathrm{Ng}$, Diagnostic markers of infection in neonates, Arch Dis Child Fetal Neonatal Ed 2004;89:F229-F235

16. Sung Youn Lee et al. Relationship between Maternal Serum C-Reactive Protein, Funisitis and Early-Onset Neonatal Sepsis, J Korean Med Sci. 2012 June; 27(6): 674-680.

17. Thomas Popovski et al.Maternal markers for detecting early-onset neonatal infection and chorioamnionitis in cases of premature rupture of membranes at or after 34 weeks of gestation: a two-center prospective study. BMC Pregnancy and Childbirth,11 Volume,2011

18. Park KH, Kim SN, Oh KJ, Lee SY, Jeong EH, Ryu Noninvasive Prediction of Intra-Amniotic Infection and/or Inflammation in Preterm Premature Rupture of Membranes A Reprod Sci 658-65 192012

19. Jantien L. van der Heyden et al.Is It Useful to Measure C-Reactive Protein and Leukocytes in Patients with Prelabor Rupture of Membranes? Amer J Perinatol 2010; 27(7): 543-547

20. Buhimschi CS et al .A low vaginal "pool" amniotic fluid glucose measurement is a predictive but not a sensitive marker for infection in women with preterm premature rupture of membranes.Am J Obstet Gynecol 2006 Feb;194(2):309-16. 